\title{
Helenos: A Realistic Benchmark for Distributed Transactional Memory
}

\author{
Jan Baranowski, Paweł Kobyliński, Konrad Siek, Paweł T. Wojciechowski \\ Institute of Computing Science \\ Poznan University of Technology
}

Mar 21, 2016

\begin{abstract}
Transactional Memory (TM) is an approach to concurrency control that aims to make writing parallel programs both effective and simple. The approach is started in non-distributed multiprocessor systems, but is gaining popularity in distributed systems to synchronize tasks at large scales. Efficiency and scalability are often the key issues in TM research, so performance benchmarks are an important part of it. However, while standard TM benchmarks like the STAMP suite and STMBench7 are available and widely accepted, they do not translate well into distributed systems. Hence, the set of benchmarks usable with distributed TM systems is very limited, and must be padded with microbenchmarks, whose simplicity and artificial nature often makes them uninformative or misleading. Therefore, this paper introduces Helenos, a realistic, complex, and comprehensive distributed TM benchmark based on the problem of the Facebook inbox, an application of the Cassandra distributed store.
\end{abstract}

\section{Introduction}

Transactional Memory (TM) [8] is an approach to concurrency control that aims to make writing parallel programs both effective and simple. The programmer applies the transaction abstraction to denote sections of code whose atomicity must be preserved. The TM system is then responsible for enacting the required guarantees, and doing so efficiently. Efficiency is often the key question in TM research, and various TM systems employ different concurrency control algorithms that can achieve quite divergent levels of performance depending on the workload. Hence, there is a need for empirical evaluation of their performance and the trade-offs between efficiency and features.

Initially, TMs were evaluated using microbenchmarks, but these test specific features in isolation and use data structures that are too trivial to draw general conclusions about a TM. Alternatively, there are HPC benchmark suites, but these are difficult to translate meaningfully into the transactional idiom. That is, benchmarks from SPEComp [2] or SPLASH-2 [24] are already expertly optimized to minimize synchronization, so any incorporated transactions are used rarely and have little effect on overall performance. Hence, a set of TM-specific benchmarks was needed, whose transactional characteristics and contention for shared resources were both varied and controllable. Thus, benchmarks and benchmark suites like STMBench7 [7], LeeTM [1], and STAMP [13] were developed.

Given that distributed systems face the same synchronization problems as their multiprocessor counterparts, distributed transactions are successfully used wherever requirements for strong consistency meet wide-area distribution, e.g., in Google's Percolator [15] and Spanner [4]. Distributed TM adds additional flexibility on top of distributed transactions and allows to lay the groundwork for well-performing middleware with concurrency control. However, in such environments, unlike its non-distributed analogue, distributed TM must also deal with a flood of additional problems like fault tolerance and scalability in the face of geo-distribution or heterogeneity.

Distributed TMs were developed by using transactions on top of replication [3, 5, 11, 9] or by allowing clients to atomically access shared resources or to atomically execute remote code [18, 22, 19]. The latter type are of particular interest and the main focus of this paper, since they incorporate the distributed elements directly into the transaction 
abstraction and apply to a wide variety of distributed system models from cluster computing to clouds and web services.

As with non-distributed TMs, the variety of differently-featured distributed TMs require empirical evaluation to find how their features, the workloads, and the configuration of distributed systems influences their performance. Therefore, as with non-distributed TMs, they must be evaluated empirically. However, the existing TM benchmarks are not appropriate for distributed TMs. This is primarily the case since the structures they use are not easy to distribute. Distributing non-distributed TM benchmarks often leads to arbitrary sharding of the structure that has no purpose for the application itself (e.g., the clients still must access the entire domain). Hence distributing STMBench7, LeeTM, or labyrinth or k-means from STAMP creates applications that do not reflect realistic use cases for distributed systems. On the other hand, even if a benchmark has valid distributed variants, the conversion is often non-trivial and should not be expected to be done ad hoc, if it is to be uniformly applied by various research teams.

As a result systems like HyFlow [18], HyFlow2 [22], and Atomic RMI [19] are all evaluated using a few microbenchmarks supplemented by a distributed version of the vacation benchmark from STAMP, which originally mimics a distributed database use case. In effect, the results of the evaluation are not always satisfactory because of the simplicity of the testing procedure. There is also little research showing comparisons between the performance of distributed TMs. Hence, in this paper we introduce Helenos, a new complex benchmark dedicated for distributed TMs, that would help to remedy the lack of tools for evaluating such systems. The benchmark proposed here is also the first step towards a creation of a comprehensive framework for evaluating all facets of distributed TMs.

The usefulness of the Helenos benchmark is demonstrated by an analysis of an example evaluation of four different distributed concurrency control mechanisms and the produced results. The aforementioned absence of other benchmarks that can fit a similar role makes conducting a straightforward comparison between Helenos and its competitors impossible. Thus, the only way for us to showcase Helenos is to perform an example evaluation presented in this paper and show that the obtained results are understandable, predictable and explicable, as well as meaningful.

This paper is structured as follows. Section 2 discusses the existing TM benchmarks in more detail and describes how they can be used for evaluating distributed TMs or what prevents them from being practical for that aim. Section 3 introduces the Helenos benchmark and provides detail as to its data model, executed transactions, and defined metrics. Then, in Section 4 we evaluate two distributed TMs and two lock-based distributed concurrency control mechanisms. Finally, we conclude in Section 5

\section{Related Work}

There are a number of TM benchmarks for non-distributed TM systems that are noteworthy. We concentrate on the ones used more often (STAMP and STMBench7), but we also present some less known benchmarks further below. We also give our attention to the benchmarks bundled with HyFlow.

\subsection{STAMP}

The most prevalent suite of benchmarks in use with non-distributed TM is the STAMP benchmark introduced in [13] and retrofitted to work with more modern TM systems in [16]. It consists of eight applications, each presenting a different algorithm, as a whole providing a wide range of transaction characteristics.

Out of the eight benchmarks within STAMP, only some can be used as distributed applications. The best candidate for a distributed benchmark is vacation. It simulates a travel agency application with a database of hotels, cars, and flights, where a number of clients attempt to book one of each, while offers are sporadically modified. The database is homogeneous in nature and can easily be distributed without incurring major modifications on the benchmark. Hence, the benchmark is often used to evaluate distributed TMs [19. 14]. However, the benchmark has a limited range of transaction types, so it does not constitute a comprehensive evaluation tool.

Other benchmarks which lend themselves to distribution are genome, bayes, ssca2, yada, and intruder. The applications are based respectively on algorithms for gene sequencing, learning structures of Bayesian networks, 
creating efficient graph representations, Delaunay mesh refinement, and detecting intrusions in a network. The processing in each of these applications follows a pipeline where a complex data structure is processed into another form in a series of steps by multiple simultaneous threads. Such processing can be distributed among several network nodes (e.g. in high performance clusters) in order to provide more system resources (processing power, memory) to the algorithms. The issue with producing such distributed versions of these algorithms is that it cannot be done ad $h o c$, and often requires that an expert-prepared variant exists with at least a reference distributed implementation that could be simply instrumented by TM researchers. In any case, from the perspective of distributed system architecture genome, bayes, ssca2, yada, and intruder are all an example of a single use-case, and therefore even though these applications provide breadth for a concurrent benchmark, they provide little breadth in the distributed context.

The kmeans benchmark represents a K-means clusterer known from data mining. The algorithm executes in rounds: in every round a thread reads the values of some partition of objects and designates one of them the new center of the cluster, then all the data objects are reassigned to the closest center. While the data for the clusterer can be distributed onto multiple nodes, the resulting variant does not present a genuine distributed use case, because execution in rounds induces too high a level of coordination required among client threads. We find the remaining labyrinth benchmark to be a similar case. There, transactions operate on a central data structure representing a 3D maze and attempt to route a path from one point to another using Lee's algorithm. Since a path cannot intersect other paths, conflicting writes must be avoided. Such an application can be employed for circuit-board design. However, even if the maze is distributed, as with kmeans, the application has no reflection in distributed system use cases.

\subsection{STMBench7}

The other popular STM benchmark, STMBench7 [7], is based on an object-oriented database benchmark. In STMBench7 clients perform a wide range of transactions on a shared tree-based database. The tree, called a module, contains three levels of nodes: a) complex assemblies ( $C A s)$, whose children are either other complex assemblies or base assemblies, $b$ ) base assemblies (BAs), which link to several composite parts, or $c$ ) composite parts (CPs), the leafs of the tree whose payload is a document and a graph. Each element contains links to its parent as well as children, allowing bottom-up or top-down traversal. Transactions in STMBench7 are either traversals (accessing a path from root to leaf), queries (accessing sets of random nodes), or structure modifications (adding or removing parts of a tree). They can be either long or short, and either read-only or update. The benchmark simulates a CAD/CAM/CASE application or a multi-user server.

The benchmark can be used for distributed TMs if the tree structure is spread among several servers in a network. This can be done in one of three ways: The benchmark can be distributed per $\mathrm{CP}$, so that each $\mathrm{CP}$ is located on the same network node as its children, but it can be located on a different server than its parent base assembly, which, in turn, can be located somewhere different than its own parent complex assembly, etc. From the perspective of the client this leads to a system with a flat structure, where the tree structure from the original application becomes a simple collection of distributed objects with some objects referring to other ones. From the distributed TM's point of view the difference between CPs, BAs, and CAs largely disappears, since their internal workings will be largely obscured. It is another practical consequence of this, that the difference between queries and traversals becomes blurred, since the only distinguishing feature between them now is how the access sets of transactions are selected (from other object in traversals rather than from an index in queries). In effect, this variant creates a very simple benchmark, which resembles the bank microbenchmark used with distributed TMs, only with a new transaction type-structure modifications.

In addition, the benchmark can be distributed per CA or BA, i.e., a BA and its children are located on the same node, but any two BAs or CAs can be located in different parts of the network. This variant allows groups of objects to be treated as a single structure by the clients, and, rather than accessing each object individually, transactions can treat a BA with all its children jointly. However, from the point of view of a distributed TM, this again becomes a flat distributed collection of remote objects. In addition to the former variant, however, the benchmark is further simplified, because the number of interdependencies between objects is smaller. This is not offset by the increased complexity of remote objects, which simply means that processing time of an access increases, but does not have any other effect on transactional processing. 
Finally, the benchmark can be distributed by adding new modules and distributing per module. However, as the authors themselves note in [7], increasing the number of modules (or distributing by module) isolates transactions from one another, and is therefore not useful for evaluating TMs. Neither of the three variants provide a satisfactory tool for evaluating distributed TMs, since distribution leads to simplification and divorcement from the original realistic CAM/CAD/CASE use case.

\subsection{HyFlow Benchmarks}

The microbenchmarks and benchmarks included by the authors of HyFlow [18] in their implementation represent what can be considered to be the best available set of benchmarks for evaluating distributed TM systems. The suite consists of three microbenchmarks, bank, loan, and distributed hashtable (DHT), as well as a distributed version of the vacation benchmark from STAMP (described above). DHT is a micro-benchmark where each server node acts as a shard of a distributed key-value store. Transactions atomically perform a number of writes or a number of reads on some subset of nodes. The bank benchmark simulates a straightforward distributed application using the bank metaphor. Each node hosts a number of bank accounts that can be accessed remotely by clients who perform transfers between accounts or atomic reads of several accounts.

Finally, the loan benchmark presents a more complex application where the execution of transactional code is also distributed among several nodes. Each server hosts a number of remote objects that allow write and read operations. Each client transaction atomically executes two reads or two writes on two objects. When a read or write method is invoked on a remote object, then it also executes two reads or writes (respectively) on two other remote objects. This recursion continues until it reaches a specified depth. Hence, the benchmark is characterized by long transactions and high contention, as well as relatively high network congestion, and is unique in focusing on the control flow transactional model.

However, while the benchmark suite is able to shed light on the performance of distributed TMs and includes a distributed TM-specific applications, it lacks complex benchmarks and therefore does not comprise a comprehensive evaluation tool.

\subsection{Other TM Benchmarks}

A number of other applications were used to test TM systems apart from the ones mentioned above. Notably, LeeTM [1] is an independent implementation of Lee's algorithm, analogous to the one used in STAMP's labyrinth. The benchmark has limited use in distributed TM evaluation for the same reasons as its STAMP counterpart. EigenBench [10] is a comprehensive and highly configurable microbenchmark for multiprocessor TM evaluation. One of its interesting features is that it allows induction of problematic executions (e.g. convoying) to observe a TM's behavior in their presence. EigenBench uses a number of flat arrays as its data structure, so it is easy to distribute for use with distributed TM. However, EigenBench is designed to test individual characteristics of a TM, and as such does not generate complex workloads containing heterogenous transactions. Another interesting application is Atomic Quake [25], a transactional implementation of a multithreaded game server, which was used to compare the performance of TM with locking in a complex realistic use case. The benchmark uses a central server to coordinate remote clients, whose nature prevents the application from being employed as a truly distributed benchmark. Finally, in [11] the authors present a custom benchmark application for replicated transactional memory called Twitter Clone which simulates the operation of a social networking service. The benchmark is not useful for non-replicated TM, but the overall idea influenced the benchmark presented in this paper.

\section{The Helenos Benchmark}

We introduce the Helenos benchmark which provides a platform for comprehensively evaluating a distributed TMs for use in non-uniform large scale distributed systems. Such a benchmark must contain a wide variety of transaction types and provide a high level of control over the workload that a TM can be subjected to. This allows thoroughly to test all aspects of a TM, and observe its performance in diverse environments. However, it is equally important that 
a benchmark be evaluated in a realistic setting, i.e., such where there exists a need for both transactional memory and for distributed systems. The benchmark is implemented in Java, which allows us to interface with some of the existing distributed TM systems either also implemented in Java [18, 19] or other languages on the Java Virtual Machine [22]. Places where transactions start and end must be manually marked for each of the tested libraries allowing great flexibility of measurement.

In order to achieve our goal of providing a realistic use case for a distributed TM, we base our benchmark on Cassandra [12], a distributed storage system for large volumes of structured data that emphasizes decentralization, scalability, and reliability. Cassandra was created to serve as a storage system for the user-to-user message inbox in Facebook, where it must be able to withstand large write-heavy throughputs. The authors specify a data model that is used for Cassandra's intended application, as well as two typical search procedures: term search (find a message by keywords) and interaction search (find all messages exchanged between two users). Cassandra allows to control consistency of its operations by managing which replicas reply to certain requests. However, the consistency guarantees can be further extended by introducing the transaction abstraction. Thus, the Cassandra implementation of the inbox is both an inherently distributed application, as well as one which can benefit from using the TM.

Hence, we use Cassandra's application as the Facebook inbox as the basis for our benchmark for distributed TM. We do this by implementing the data model specified in [12] and supplement the original search procedures with a comprehensive spectrum of pertinent transactions. On the other hand, we remove features that are not directly á propos, including fault tolerance, local persistence, and partial replication. This makes any evaluation results simpler to predict and analyze, but does so without loosing the nuance of the original benchmark.

\subsection{Data Model}

We follow Cassandra's data model and a logical data model from the inbox application in [12]. First of all, a Cassandra cluster is composed of several nodes forming a logical topology of a ring. Each server node being assigned an arbitrary (random) position in the ring. The dataset is distributed among these nodes by deriving a position in the ring from a hash of each data item's key, and assigning that item to the first node with a greater position. In this way the data items are "wound around" the ring (multiple times) and one node becomes responsible for multiple ranges of keys. We refer to each such range as a bucket and use this level of granularity for synchronization. That is, two transactions conflict if they try to access the same bucket. We show this model in Fig. 1.

The original inbox application data model consists of two tables distributed in a Cassandra cluster, one for term search and one for interaction search. Each table row is a separate data item containing data from all of the table's columns in that row (as the object's fields). The users of the application all have unique identifiers (UserID), as do all messages in the system (MsgID). Note that the data resolution changes between the original application and the benchmark, since bucket-level granularity gives us a greater level of control over contention.

The term search table (TermTable) is used to find messages in a user's inbox by keywords. The logical structure of the table consists of one column containing a UserID and one column for each possible keyword. Each keyword column contains lists of message identifiers (MsgIDs) of messages that include this keyword. More formally:

$$
\text { TermTable : UserID } \mapsto \text { Keyword } \times(\text { MsgID list) }
$$

This schema assumes that the number of users is orders of magnitude greater than the size of one user's inbox. However, this is problematic for the purposes of a benchmark, since it makes contention both lower and more difficult to adjust. Hence, we decided to modify this table to increase the number of keys by moving the keyword data from separate columns into the key. In effect, the number of objects per user is increased and contention is no longer strictly dependent on the number of users but can be controlled by adjusting the population of keywords. Hence our schema looks as follows:

$$
\text { TermTable : UserID } \times \text { Keyword } \mapsto(\text { MsgID list })
$$

Note that, since the benchmark only stores hashes of keys, the hashed keyword information has to be kept elsewhere in addition to the key column, but we omit this detail for clarity. 


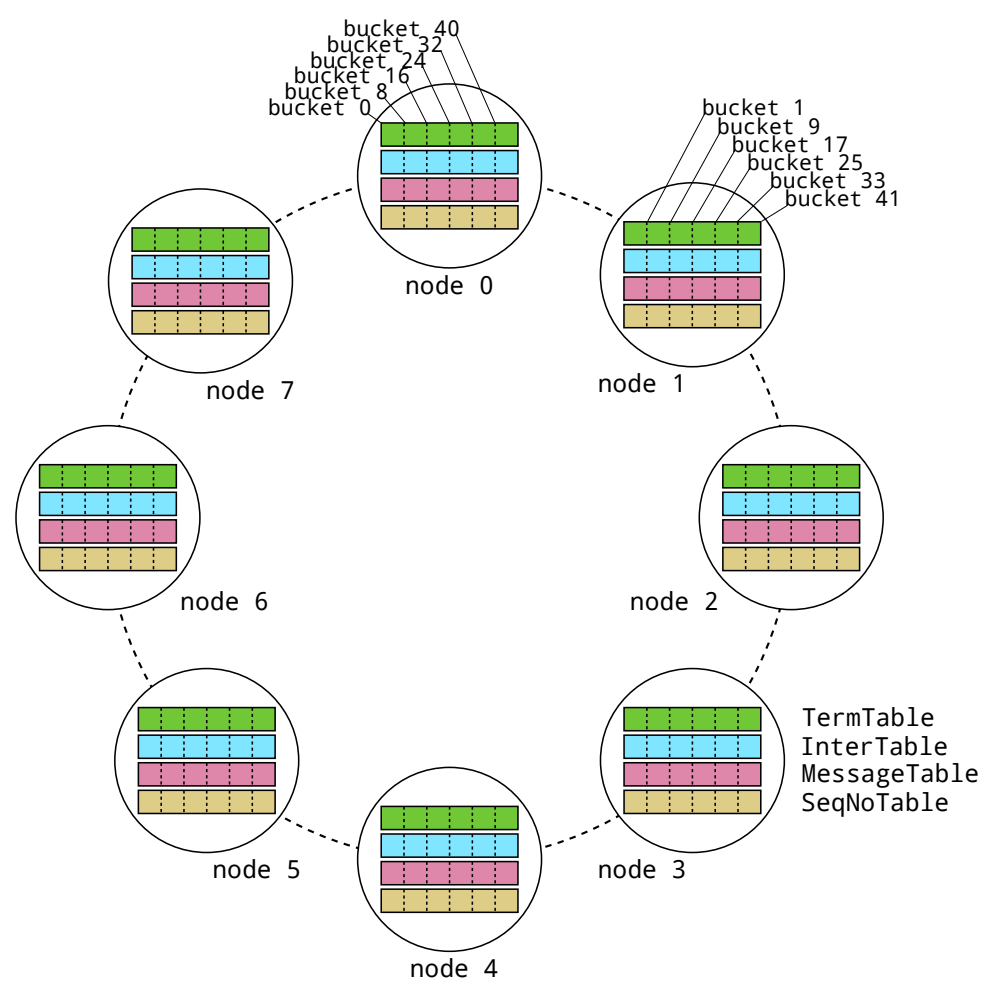

Figure 1: Database architecture.

The interaction search table (InterTable) stores conversations between users. The table's key is the identifier of the user sending the message. The table also contains one column per receiving user which holds the IDs of the messages exchanged between the two users. Thus:

$$
\text { InterTable : UserID } \mapsto \text { UserID } \times(\text { MsgID list })
$$

This schema encounters the same problems as TermTable, so we perform a similar modification in order to increase the key domain: we use an ordered pair indicating the sender and the receiver of the message as the key, rather than just the sender. Thus, in the benchmark the table is defined as:

$$
\text { InterTable : UserID } \times \text { UserID } \mapsto(\text { MsgID list })
$$

We extend the data model by adding additional tables which can be used to introduce new functionality into the application, and in this way increase the benchmark's depth — widen the range of transactions that can be performed. The message table (MessageTable) stores the contents of the messages used in the system (note that TermTable and InterTable both operate on message identifiers alone). The table's key is the identifier of the recipient of the message, and the value is the message, which can be expressed as five columns: the identifier of the message, the user identifiers of the sender and the recipient, the text of the message, and the timestamp of when it was sent. (The timestamps are used to sort messages, allowing transactions to return a group of most recent ones). In effect, we have the following definition:

$$
\text { MessageTable }: \text { UserID } \mapsto(\text { MsgID } \times \text { UserID } \times \text { UserID } \times \text { Text } \times \text { Time }) \text { list }
$$

Finally, we employ a sequential number table (SeqNoTable) which is used to generate new message identifiers for a given user's inbox and to specify a cut off point for deleting old messages. Thus, the table's key is the identifier 


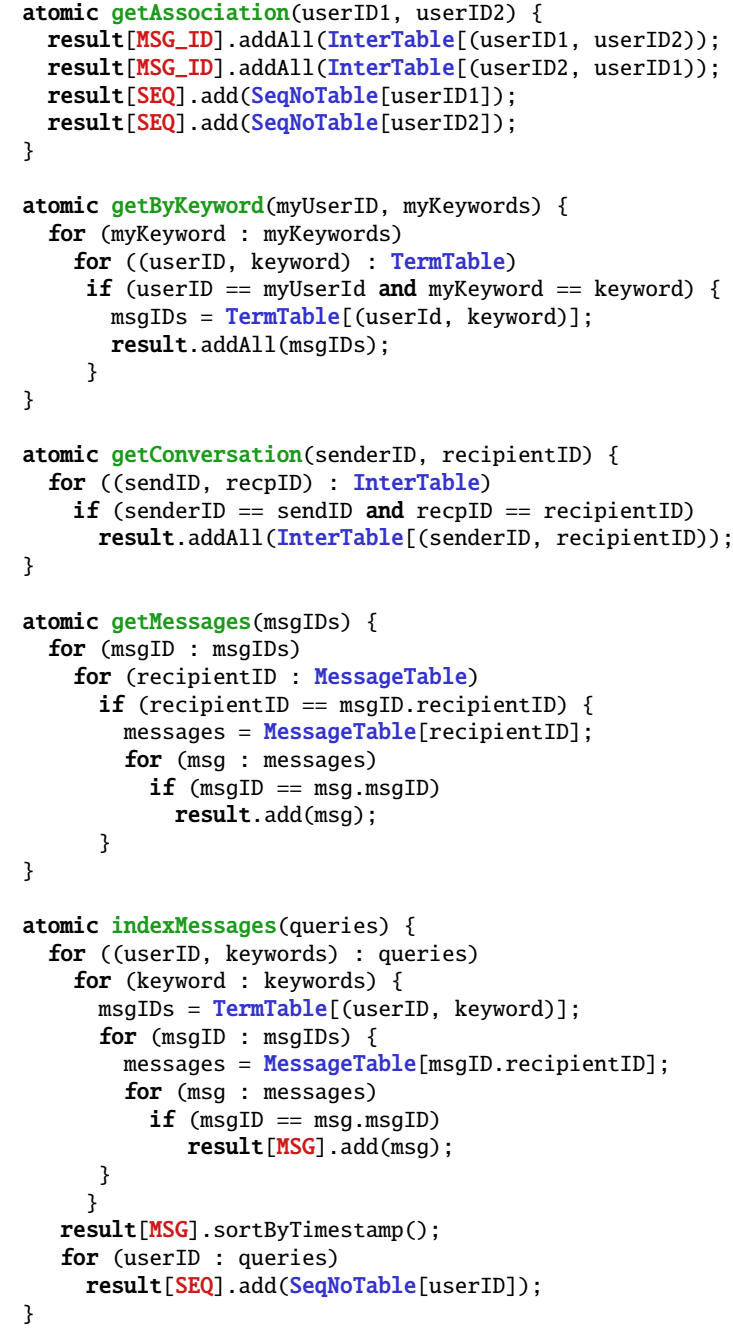

Figure 2: Benchmark transactions pseudocode.

of an owner of an inbox and the data columns include the sequence number (SequenceNo) of the most recently created message in the inbox and the sequence number of the last deleted message there. This is defined as:

\section{SeqNoTable : UserID $\mapsto$ SequenceNo $\times$ SequenceNo}

\subsection{Tasks and Transactions}

Clients in the benchmark can run 8 types of high-level tasks on the distributed database. Each of these tasks performs some non-transactional operations (primarily locating nodes in the ring from hashes and performing local processing) and employs one or more atomic transactions. The first two tasks: term search and interaction search are a part of the original example in [12], and the remainder are domain-appropriate additions that ensure the configurability of the benchmark. We describe the tasks below and provide detail about the transactions they use. All transactions are further detailed in Fig. 2. We also provide a summary in Fig. 3. 
Term Search A user (identified by a specific UserID) specifies a set of keywords. The user's inbox is then searched to find all messages which contain one of the keywords by using transaction getByKeyword. This transaction traverses TermTable and returns all message identifiers whose key both matches the user's ID and at least one of the specified keywords. This section must be atomic to return a consistent state of the inbox. Finally, the contents of the matching messages are retrieved with transaction getMessages, which retrieves the contents of the messages from MessageTable on the basis of the list of message identifier. Again, this is done atomically, in order to return a consistent snapshot of the inbox. Overall, the term search task reads from two tables: TermTable and MessageTable and we estimate it to be medium in length. The length of the task will depend on the size of the keyword domain.

Interaction Search A user specifies a UserID of another user in order to find all conversations between the two of them. In order to do this, the task runs transaction getConversation, which gets all message identifiers from InterTable whose keys fit the user identifiers of both users in question. Then, the contents of the messages are retrieved using getMessages. Thus, interactions search reads from two tables: InterTable and MessageTable and is of small length, depending on the number of users in the system and the number of messages in both the users' inboxes.

Send Unicast A user, the sender, sends a message consisting of a text content to another user, the recipient. This consists of executing transaction sendMsg which is preceded by extracting the set of keywords from the contents of the message. The transaction then increments and retrieves the next sequence number from SeqNoTable and uses it to create a new MsgID. Then, a message object is created and written into MessageTable. Subsequently, the identifier of the message is inserted into InterTable twice: once to indicate the message sent out from the senders inbox, and once to indicate the message received in the inbox of the recipient. Finally, the transaction inserts an entry into TermTable for each keyword extracted from the contents of the message. All these actions must be performed atomically, in order to prevent another transaction from interfering and causing an inconsistent state. E.g., if a message were removed by another transaction from the MessageTable while this task was yet to add all the keywords to TermTable, the contents of TermTable would point to messages which no longer existed. Overall, this task updates SeqNoTable and writes to all the other tables. On the whole, this is a short read/write task with a relatively limited read/write set (R/W set).

Send Multicast A user sends a single message to several recipients. This task is an extension of the send unicast task (defined above), where the sendMsg transaction is executed in series, but the entire series is executed atomically. This task is a medium- to large-sized write task that touches the same tables as the send unicast task, but it strongly depends on the number of recipients.

Batch Import The system is given a set of complete messages to store in the database. This represents a use case where the database is replicated and two of the replicas synchronize, by one sending a state update to the other. Another pertinent scenario is one where the database tries to recover from a crash. The task involves extracting keywords from each message's contents and executing transaction importMessages. The transaction filters the set of imported messages against SeqNoTable to remove all those that have a sequence number lower or equal to the sequence number of the last deleted message. If a message has a higher sequence number than the current highest sequence number in SeqNoTable, then the database is also updated. Then, the transaction filters out those messages which are already in MessageTable. All the remaining messages are added to MessageTable, InterTable, and TermTable by analogy to sendMsg. The task is a long write task (depending on the number of imported messages) which updates SeqNoTable and MessageTable, and writes to the two remaining tables.

Clear Inbox A user requests that all messages from her inbox be removed. This task sequentially executes three separate transactions. First, the task runs transaction resetCutoff which retrieves the sequence numbers of the most recent message in the user's inbox as well as the sequence number of the most recent deleted message from SeqNoTable. In addition, the transaction sets the deleted sequence number to the current sequence number, signifying that all messages in the inbox are now below the cutoff for deletion. Next, the task uses transaction getMessages 


\begin{tabular}{|l|l|l|l|}
\hline Task & R/W set & Length & Used transactions \\
\hline \hline association level & read-only & short & getAssociation \\
term search & read-only & medium & getByKeyword, getMessages \\
interaction search & read-only & short & getConversation, getMessages \\
indexing & read-only & long & indexMessages \\
send unicast & read/write & short & sendMsg \\
send multicast & read/write & medium/long & sendMsg \\
batch import & read/write & long & importMessages \\
clear inbox & read/write & medium & resetCutoff, getMessages, removeMessages \\
\hline \hline Transaction & R/W set & Length & Touched tables \\
\hline \hline getByKeyword & read-only & short/medium & TermTable \\
getConversation & read-only & short & InterTable \\
getAssociation & read-only & short & InterTable \\
getMessages & read-only & medium & MessageTable \\
indexMessages & read-only & long & TermTable, SeqNoTable \\
resetCutoff & read/write & short & SeqNoTable \\
sendMsg & read/write & medium & TermTable, InterTable, MessageTable, SeqNoTable \\
importMessages & read/write & long & TermTable, InterTable, MessageTable, SeqNoTable \\
removeMessages & read/write & medium & TermTable, InterTable, MessageTable \\
\hline
\end{tabular}

Figure 3: Overview of tasks and transactions in Helenos.

to retrieve all the existing messages in the inbox. Afterward, the task executes transaction removeMessages to remove all the messages from the three tables in the database that hold message information. Specifically, for each keyword of each message to be removed, the transaction removes each message's identifier from TermTable. Then, on the basis of the recipient and sender of each message, the transaction removes both occurrences of its message identifier from InterTable. Finally, the transaction searches each message in MessageTable by their recipient and remove them from there. The task is a medium-sized read/write tasks (although the execution can be shorter depending on the number of messages in the inbox) and touches all tables. The task is not executed atomically as a whole because any potential inconsistencies stemming from the lack of atomicity between transactions can be fully and easily resolved by the application. Thus this decomposition makes for a more realistic workload.

Association Level Given two users, the system checks what is the level of interaction between them, by counting the number of exchanged messages and normalizing the number against the number of messages in the inbox. This involves running transaction getAssociation, which retrieves the identifiers of messages involved in conversations between both users (from InterTable). It also retrieves the sequence numbers of the most recent existing and the most recent deleted message in both users' inboxes (from SeqNoTable). The results of these executions are then processed non-transactionally by the task. This produces a short read-only task operating on a single tableInterTable. We add this task to have a finer control over contention, since the existing short read-only transactions in interaction search and term search are always followed by a medium-sized read-only transaction.

Indexing Creates a cache for the most common keyword searches by the most active users. Given a list of users and a list of keyword search queries per user the task runs transaction indexMessages. For each user and for every keyword, the transaction searches through TermTable and collects message identifiers of all pertinent messages. Then, unique message identifiers are extracted from the list. The results are then sorted and cropped to a prescribed length. Then, the transaction retrieves the body of the three example messages for each user from MessageTable. Finally, the transaction retrieves sequence number information from SeqNoTable for each of the investigated users. Indexing is a work-intensive read-only task that contains a long transaction with a large readset. 


\subsection{Metrics}

In recognition of the fact that complex systems require comprehensive metrics, the benchmark implementation includes several metrics useful for monitoring distributed TM performance.

Classically, we provide the commonly employed throughput metric, defined as the number of transactions executed per second by the system. We also measure latency (or mean flow time), which is an alternative performance goal to throughput. A single transaction's latency (flow time) is the time between a transaction commences and finally completes. This time includes any time spent re-executing the transaction due to forced aborts. The system's metric is the mean of all transactions' latency. We measure latency on the system level as well as per transaction. Furthermore, we allow measuring transactions' abort rate, which indicates how often transactions are forced to re-execute. These metrics allow to measure to what extent a TM's overall performance goals were reached. They also constitute the most basic tool for comparing different TMs.

In addition, we provide more diagnostic metrics that allow to reason about the workload itself. First of all, we measure retry rate, total execution time of all transactions, total execution time of all retries, sum of all startup times for all transactions i.e. sum of all periods of time between the start of a transaction and the start of the first retry of this transaction, as well as total number of operations on all the buckets. Finally, we supplement these measures with metrics showing the total execution time of the system, total parallel execution time i.e. time between the start of the first client thread and the end of the last client thread, and transactional execution ratio, the percentage of time the system spent within transactions.

\subsection{Parameters}

The benchmark includes a number of parameters that can be used to evaluate a TM in a range of workload types. The most important aspect controlled by the parameters is contention, which can be controlled by adjusting the number of shared objects in relation to the number of transactions that use these objects on average, and to the number of objects used by each transaction. Larger R/W sets or more clients in relation to the same number of objects means increased contention.

We allow fine control over the domain of shared objects by changing the configuration of the system: the number of network nodes, and the number of remote objects (buckets) per table. We also allow changing the domain of words allowed in messages, which increases the number of keys in TermTable. The number of transactions is controlled by adjusting the number of simultaneous clients in the system, as well as the number of tasks executed by each of them. Finally, the R/W sets of transactions can be controlled directly by adjusting the cap on the number of keywords allowed in a query (getByKeyword, indexMessages), setting the minimum and maximum number of messages for importMessages.

The benchmarks also allow to control the composition of the workload, by specifying what percentage of which types of transactions will be executed. This allows to test various features of TM memories that depend on e.g., a high ratio of read operation or read-only transactions. In addition, since moving data around a network can have a significant cost, we allow adjusting the size of data by setting the maximum length of the content of messages in MessageTable. Hence caching and storage strategies used by various distributed TMs can be evaluated in various environments. Lastly, we introduced a configurable delay in milliseconds that is applied to every operation on every bucket in the system. It can be used to mimic higher network latency or slower nodes.

\section{Evaluation}

In this section we present an example of empirical evaluation of several distributed concurrency control mechanisms, including two distributed TMs representative of two divergent approacches to synchronization, using Helenos. We must stress, however, that the purpose of the paper is not to provide a comprehensive evaluation of the TM systems in question (both of which were evaluated in their repsectve papers [21, 22]). Instead, the main objective of this evaluation is to provide a set of reference parameters and workloads and demonstarte how particular settings impact the benchmark's workload and the performance of disparate concurrency control schemes. In effect, this shows that Helenos' parameters are impactful and that the benchmark can be used to mimic complex, real-life distributed 


\begin{tabular}{|l|c|c|c|c|c|c|c|}
\hline Task type & Standard & Small R & Small R/W & Small W & Large R & Large R/W & Large W \\
\hline \hline term search & 0.25 & 0.30 & 0.19 & 0.02 & 0.44 & 0.19 & 0.02 \\
interaction search & 0.20 & 0.30 & 0.19 & 0.02 & 0.02 & 0.02 & 0.02 \\
send unicast & 0.06 & 0.02 & 0.19 & 0.44 & 0.02 & 0.02 & 0.02 \\
send multicast & 0.04 & 0.02 & 0.02 & 0.02 & 0.02 & 0.19 & 0.30 \\
batch import & 0.04 & 0.02 & 0.02 & 0.02 & 0.02 & 0.18 & 0.30 \\
clear inbox & 0.06 & 0.02 & 0.18 & 0.44 & 0.02 & 0.19 & 0.30 \\
association level & 0.20 & 0.30 & 0.19 & 0.02 & 0.02 & 0.02 & 0.02 \\
indexing & 0.15 & 0.02 & 0.02 & 0.02 & 0.44 & 0.19 & 0.02 \\
\hline
\end{tabular}

Figure 4: Task type probabilities in evaluation scenarios.

systems. In addition, we showcase how an evaluation can be used to draw meaningful diagnostic conclusions and provide a reference benchmark for future research in distributed TM.

\subsection{Frameworks}

For the purpose of comparison we use four distributed concurrency control frameworks, including two distributed TM implementations that represent two main approaches to transactional synchronization, and two typical distributed locking schemes.

The first distributed TM we evaluate is HyFlow2 [22], a state-of-the-art optimistic data-flow distributed TM implemented in Scala. The optimistic approach to concurrency control means that when two transactions conflict on a shared object, one of them is aborted and re-executed. The data-flow model means that whenever a client accesses a shared object, the object is moved to the client's node for the duration of the access (but there is always exactly one copy of each object in the system). HyFlow2 implements the Transaction Forwarding Algorithm (TFA) [17] to handle synchronization (with the guarantee of opacity [6]) and uses the Akka library for networking. For the evaluation we configure HyFlow2 to use standard Java serialization.

The second distributed TM is Atomic RMI [21], a pessimistic control-flow distributed TM implemented in Java, on top of Java RMI. The pessimistic approach means transactions defer operations to avoid conflicts altogether and thus preventing transactions from aborting. The control flow model means that shared objects are immobile and execute any code related to accesses on the nodes they are on. Atomic RMI uses the Supremum Versioning Algorithm (SVA) [23] for concurrency control, which guarantees last-use opacity [20].

The two locking schemes used for the evaluation are fine grained locks (FGL) and a global lock (GLock). FGL simulate an expert implementation of a distributed locking scheme using mutual exclusion locks with one lock per shared object. Locks in FGL are always acquired and released according to two-phase locking (2PL) and used according to a predefined global locking order to prevent deadlocks. Locks are released in FGL after the last access to a particular object within a given transaction. Such an implementation of 2PL is trivially last-use opaque. GLock is a locking scheme where the system contains a single lock on one of the nodes that must be acquired at the beginning of each transaction and held throughout each transaction's duration. This means that only one transaction can be executed at a time in the entire system. Hence, this is an anchor implementation, roughly equivalent to using a sequential baseline execution in non-distributed TM evaluations. GLock is trivialy opaque. Both locking schemes used for the evaluation are built on top of Java RMI and use custom lock implementations.

\subsection{Testing Environment}

We perform our evaluation using a 16-node cluster connected by a 1Gb network. Each node is equipped with two quad-core Intel Xeon L3260 processors at $2.83 \mathrm{GHz}$ with 4 GB of RAM each and runs a OpenSUSE 13.1 (kernel 3.11.10, x86_64 architecture). We use the 64-bit Java HotSpot(TM) JVM version 1.8 (build 1.8.0_25-b17). 


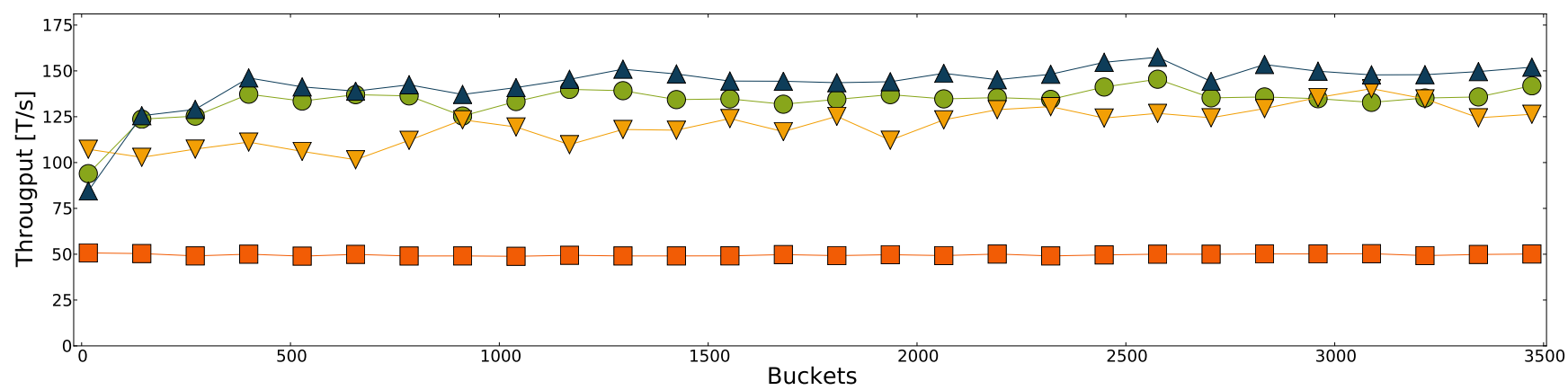

(a)

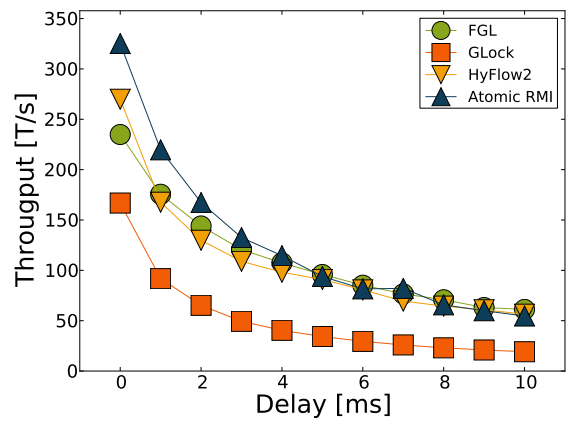

(b)

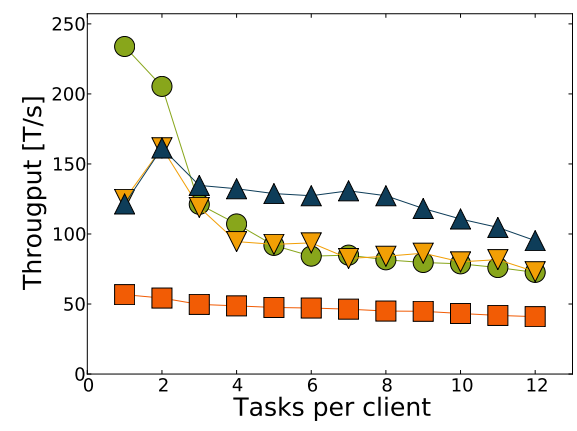

(c)

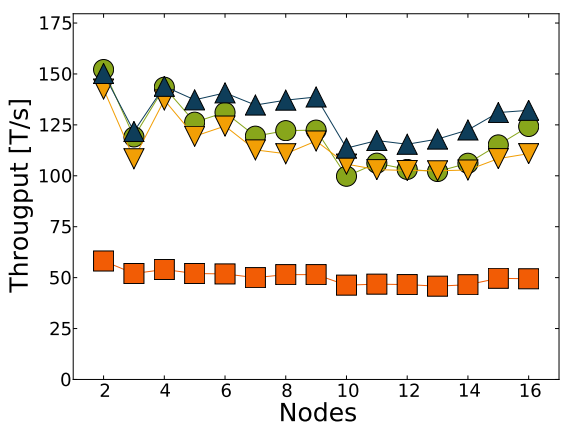

(d)

Figure 5: Impact of parameters on throughput.

\subsection{Parameter Settings}

As the first part of our evaluation we showcase the benchmark's configurability by displaying the behavior of the four frameworks within diverse workloads generated by manipulating the parameters of Helenos. The starting point for the reconfiguration is the standard workload, which has an $80-20 \%$ read-to-write-task ratio (the probabilities of executing a specific task are shown in Fig. 4 typically used for TM evaluation. The basic configuration of the standard scenario contains 1024 buckets deployed on 16 nodes, with 200 concurrent clients executing 3 consecutive tasks each. The message length is set to 8 words and the operation delay is set to $3 \mathrm{~ms}$. We treat these parameters as our reference for comparing TM implementations. The parameters were selected for the standard benchmark on the basis of experimentation to simulate a well-behaved distributed TM system. We then show how the manipulation of these reference values impacts the performance of the various concurrency control mechanisms.

Buckets Fig. 5a|shows the impact of manipulation of the number of buckets used within the system on throughput. As the number of buckets is increased, transactions are less likely to access the same bucket, so the system's contention decreases. A decrease in contention leads to an increase in throughput for all evaluated frameworks, but the change has varying impact. The evaluation shows that Atomic RMI and FGL both capitalize on higher contention in the range between 1 and 500 buckets, which is attributable to the higher costs of conflict avoidance in those frameworks. This is contrasted with HyFlow2, whose throughput increases more gradually in the same range, as contention lowers. When the number of buckets exceeds 1000, any further increase does not result in an improvement in throughput, showing that at this point contention is negligible and other factors, like operation overhead, dictate performance. Given these results, we use 1024 buckets as the reference value. 
Delay Fig. $5 \mathrm{~b}$ shows the transactional throughput of the four frameworks as the length of the operations executed by transactions changes. Naturally, as the length of each operation increases, the throughput of the system decreases (even for a sequential execution simulated by GLock). Here, all frameworks behave similarly, showing a steady decrease in performance, that converges around the $10 \mathrm{~ms}$ mark. The reference delay value we select is $3 \mathrm{~ms}$, at which point the throughput for FGL, HyFlow2, and Atomic RMI is in the vicinity of 175 transactions per second, with a slight divergence.

Tasks per client Fig. 5c shows the change in transactional throughput as the number of consecutive tasks executed by each client increases. The results clearly show anomalous behavior for low task numbers, that significantly diverges from the results for higher task values. The divergence stems from the heterogeneity of tasks in Helenos, which means that for low task values some clients will finish their tasks much faster than their counterparts that execute longer tasks at the same time. If the number of consecutive tasks is low, the clients with shorter tasks finish execution early and leave the system with a diminished number of concurrent clients, thus randomly, and unwarrantably decreasing the system's contention. When the number of tasks per client is increased, the results show that performance steadies for all frameworks. On the other hand, increasing the task count leads to a prohibitive total execution time for the entire benchmark (e.g. for FGL, the execution time for each data point is 35.2s for 3 consecutive tasks, $43.1 \mathrm{~s}$ for 4 , and $96.4 \mathrm{~s}$ for 10). Hence, we select 3 tasks, the least value where the performance is not anomalous, for the reference value.

Nodes Fig. $5 \mathrm{~d}$ shows the reaction of the four frameworks to changes in network size. As nodes are introduced into the distributed system, the contention and transactional characteristics remain constant, which causes little change in the throughput values for all frameworks. The number of nodes, along with the number of buckets, decides how buckets are distributed in the system. Given a constant number of buckets, the lower the number of nodes, the greater the number of buckets that are hosted per node. This change itself does not impact the behavior of the TM frameworks themselves, however. What the fluctuation in throughput reflects is the impact of the physical properties of the testing environment itself in general, and the network topology as well as communication costs in particular, which is consistently more pronounced for certain configurations (i.e. for a system with 3 nodes or 10 nodes). The reference value for node count selected for the benchmark is 16 , since the TM applications are meant to be used in larger rather than smaller systems, and that is the largest configuration available to us.

Message length Fig. 6 shows the impact of setting a different message length for the contents of messages, as it affects TermTable. An increasing message length has a visible effect on all those transactions that access TermTable, since it increases the access sets of transactions that read from that table (because parts of a message are distributed among a greater number of buckets) and the length of write operations on that table. Hence, increasing the message length impacts contention, and thus decreases the transactional throughput of each framework. The impact is most visible for FGL, where an increased message length causes a significant drop in the presence of high client numbers. The reference value for message length is set at 8 words.

\subsection{Scenarios}

Fig. 7 shows a comparison of the four benchmarks using different workloads, wherein clients execute different sets of tasks. The composition of particular scenarios in use is shown in Fig. 4. but generally, scenarios marked small consist primarily of short and medium tasks, with a minimal number of long tasks, whereas scenarios marked large consist primarily of medium and long tasks, with sporadic short tasks also occurring. Scenarios marked $R$ are readdominated, consisting predominantly of tasks executing read-only transactions, $W$ are write-dominated, consisting mostly of tasks executing read/write transactions, and $R / W$ scenarios are balanced. Comparing the performance of the four frameworks using a varied set of scenarios allows us to evaluate in a realistic environment and draw conclusions from their performance.

In scenario Small $R$ (Fig.7a), we see that the increase in contention driven by a growing number of clients impacts Atomic RMI least, whereas the impact on FGL and HyFlow2 is significantly more visible. HyFlow2 and FGL start 


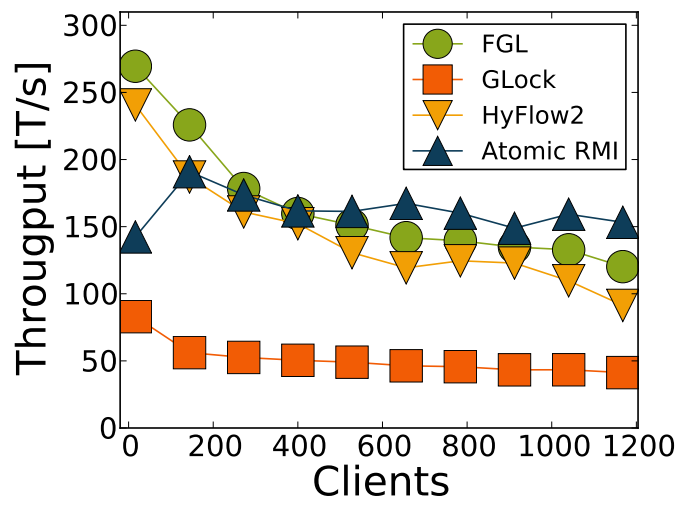

(a) Throughput with message length at 4 words.

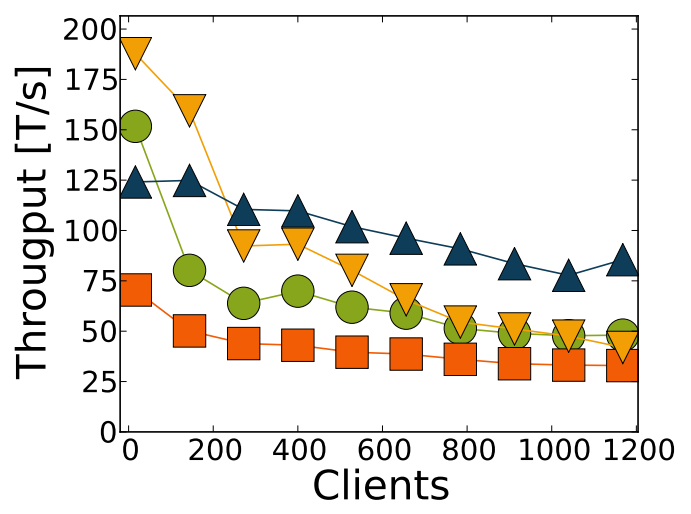

(c) Throughput with message length at 12 words.

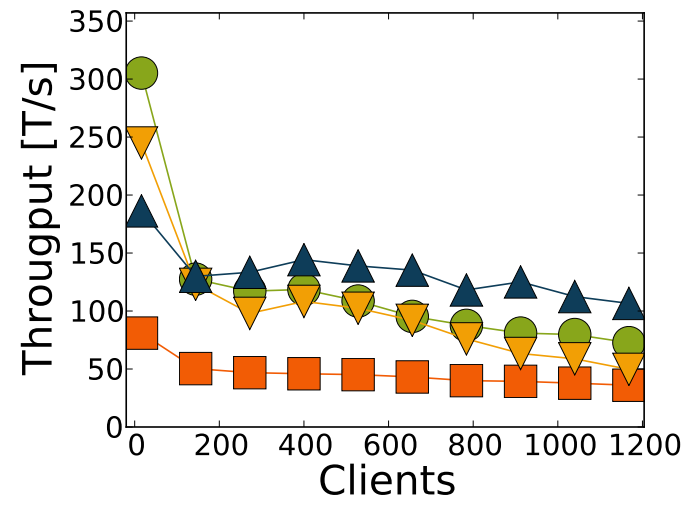

(b) Throughput with message length at 8 words.

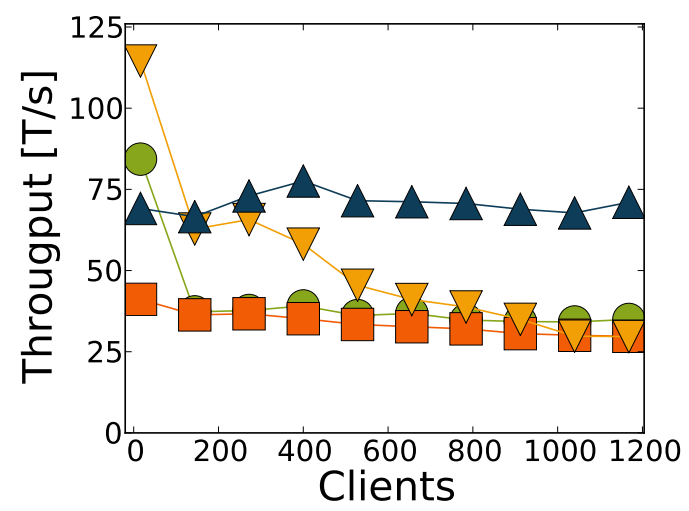

(d) Throughput with message length at 16 words.

Figure 6: Impact of message length on throughput.

off with an advantage in performance, with throughput as high as 250 transactions per second, until the number of clients reaches 200, when the performance suddenly degrades and stabilizes between 75 and 100 transactions per second for client counts larger than 300. In contrast, Atomic RMI retains a constant throughput between 100 and 125 transactions per second regardless of scale. We see, however, that the aforementioned frameworks manage to scale in the 300-1200 client range with reasonable throughput, and retain an advantage of a sequential execution of at least $25 \%$ and up to $150 \%$. The benchmark then shows that for specific sets of parameters and a particular workload Atomic RMI maintains better scalability, whereas solutions like FGL and HyFlow2 have a definite edge in lower contention environments.

In scenario Small $R / W$ (Fig. $7 \mathrm{~b}$ ) we see a general decrease in throughput, as a large contingent of read/write operations are introduced into the benchmark. These shape the transactional characteristic (e.g. for all frameworks there is a gradual degradation in performance. Fig. $7 \mathrm{~b}$ and Fig. $7 \mathrm{cc}$. Due to its operation-type agnostic concurrency control method and an automated method of early release, Atomic RMI handles the increase in write operations better than FGL and HyFlow until the contention increases significantly with the introduction at 800 clients, at which point all frameworks tend to converge and achieve a near-sequential performance (up to 30 transactions per second for Atomic RMI vs 20 transaction per second for GLock). Even though FGL is also pessimistic and has early release, releasing objects early incurs additional network messages in that scheme (since a lock has to be signaled), which, in turn, impacts performance in a highly saturated network such as the one in this scenario. On the other hand, a large number of write operations and growing contention cause HyFlow2 to achieve a significant abort rate and 


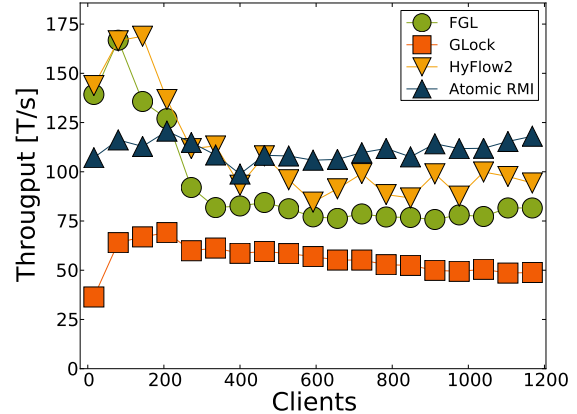

(a) Small R scenario.

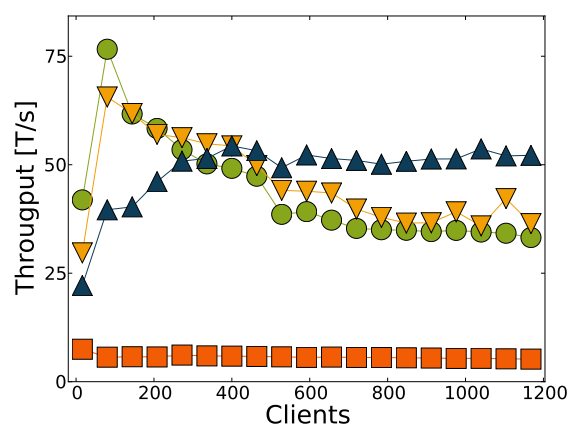

(d) Large R scenario.

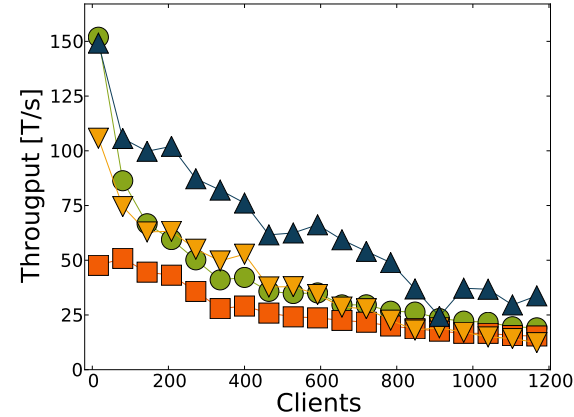

(b) Small R/W scenario.

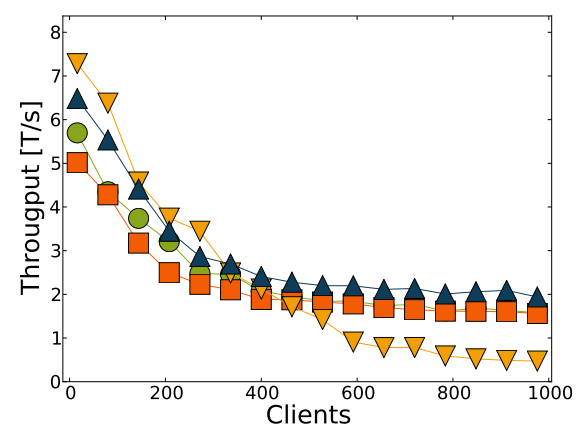

(e) Large R/W scenario.

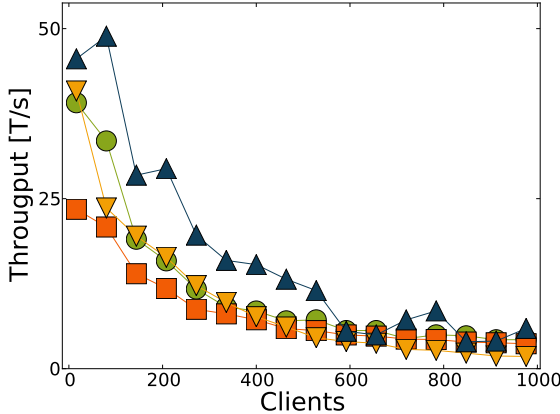

(c) Small W scenario.

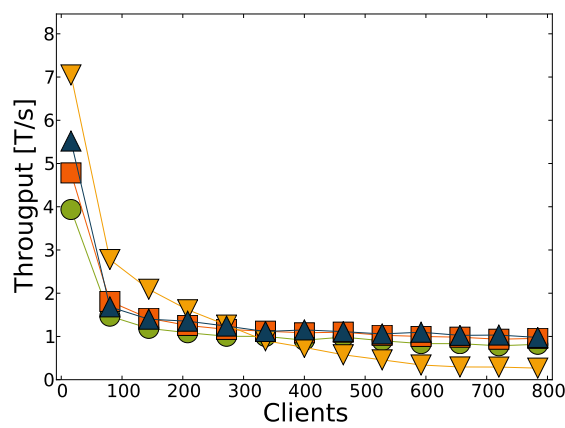

(f) Large W scenario.

Figure 7: Workload configurations.

a high retry rate (see below), causing the transactions to often waste work and incur additional network traffic as data-flow objects are supplied to transactions. The results show that pessimistic TM may be favorable to optimistic TM in a high contention write-dominated distributed system.

The Small $W$ scenario (Fig. 7C) is analogous to Small R/W, showing similar performance characteristics, but between $50 \%$ and $75 \%$ decreased. It is plainly visible that for relatively low numbers of clients (around 600 ) all frameworks tend to saturate and perform the same as or not much better than a sequential evaluation.

The Large $R$ scenario (Fig. $7 \mathrm{~d}$ ) shows similar behavior to Small $R$, but longer transactions have an impact on the performance of all four frameworks, that is especially visible in the 1-400 client range when the benchmark has relatively low contention. Note that transactional throughput values for all frameworks are nevertheless much lower than in Small R, since the transactions are much longer on average. At the outset, FGL, HyFlow2, and Atomic RMI all achieve comparatively low throughput, which increases as new clients are added. We attribute this to the initial undersaturation of the system, which means that adding new clients only increases tasks executed in parallel, without increasing the incidence of conflicts. Hence, Atomic RMI gradually increases throughput until it reaches 50 transactions per second at the 400 client mark. On the other hand, the throughput of HyFlow2 and FGL increases more rapidly with the introduction of new clients and peaks with around 100 clients at 70-75 transactions per second. This, however saturates both frameworks, and any further added clients cause a decline in performance, until both frameworks stabilize around the 600 client mark at about 30-40 transactions per second. Hence, as in Small $R$, there is range of load for which optimistic TM or a more subtle locking solution is preferable to pessimistic $\mathrm{TM}$, but the distinction is much more pronounced. On the other hand, once contention reaches a certain threshold, pessimistic TM gains a stable advantage over the other two types of frameworks. Thus, the choice of paradigm must be tailored to the application and its workload, and Helenos provides important clues as to how this adjustment should be made. 


\begin{tabular}{|c|c|c|}
\hline \# Clients & Abort ratio & Retry rate \\
\hline \hline 16 & $11.8 \%$ & 1.255 \\
80 & $22.4 \%$ & 2.900 \\
144 & $17.7 \%$ & 4.516 \\
208 & $26.1 \%$ & 6.044 \\
272 & $21.8 \%$ & 7.744 \\
336 & $30.2 \%$ & 8.625 \\
400 & $23.1 \%$ & 10.278 \\
464 & $21.5 \%$ & 12.903 \\
528 & $30.9 \%$ & 13.909 \\
592 & $29.5 \%$ & 16.805 \\
656 & $30.1 \%$ & 18.522 \\
720 & $23.3 \%$ & 20.657 \\
784 & $22.5 \%$ & 23.772 \\
848 & $21.8 \%$ & 26.812 \\
912 & $29.0 \%$ & 27.750 \\
976 & $25.9 \%$ & 31.424 \\
1040 & $26.0 \%$ & 34.406 \\
1104 & $21.8 \%$ & 36.263 \\
1168 & $26.8 \%$ & 38.932 \\
\hline
\end{tabular}

Figure 8: HyFlow2 abort ratio and retry rate for the standard scenario.

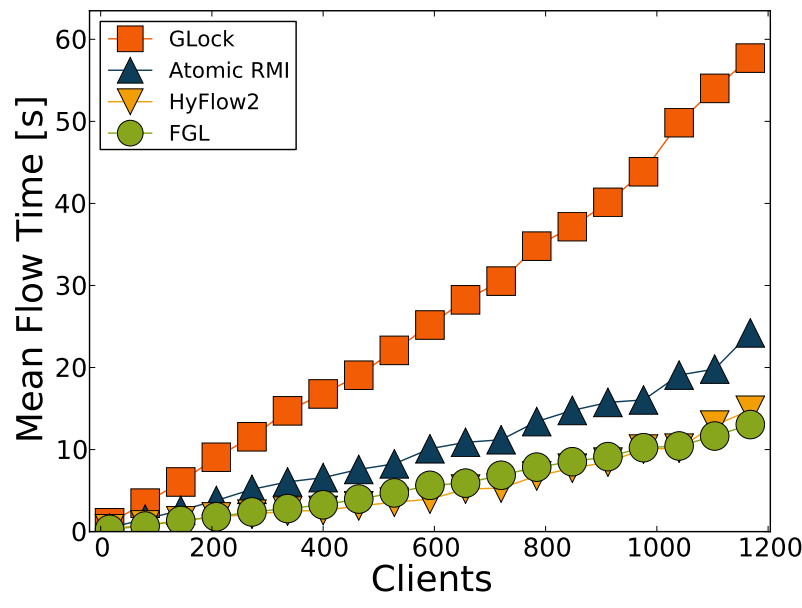

Figure 9: Mean flow time for the standard scenario.

When large read-write tasks are introduced in both Large W (Fig. $7 \mathrm{f}$ ) as well as Large R/W (Fig. 7e), they throttle the throughput of all frameworks, since they have long execution times, as well as large R/W sets, and therefore typically cannot be executed in parallel to other tasks. Hence, throughput is below 8 transactions per second even for low contention situations. Furthermore, all frameworks perform similarly to GLock with Atomic RMI performing marginally better, FGL performing the same, and HyFlow2 performing notably worse. The performance of optimistic TM below GLock is attributable to the tendency of the workload to generate a large incidence of conflicts, leading to a high abort rate $(52.5 \%$ for Large $R / W$ and $68.8 \%$ for Large $W$ ) and a hight retry rate (211 retries per commit in Large $R / W$ and 803 for Large $W$ ).

\subsection{Aborts}

We show the abort and retry rates for Fig. 8 The figure shows the results for HyFlow2, whereas the remaining frameworks had a consistent abort rate of 0 (i.e. no aborts at all) and a retry rate of 1 (i.e. one attempt to execute a transaction per commit). The results for HyFlow2 show that the abort rate of that implementation tends to fluctuate around a certain stable level, despite increasing contention with the number of clients. However, the retry rate shows, that transactions that abort tend to abort more often on average as the contention increases, leading to a high number of aborts total.

\subsection{Mean Flow Time}

The benchmark allows to perform other measurements, including mean flow time. We show an example of that measurement in Fig. 9 for the standard scenario. Mean flow time indicates, that whereas the analysis of throughputs prefer Atomic RMI over FGL and HyFlow2 in the standard scenario, the average time between a transaction's start and commitment is lower in FGL and HyFlow2. Hence, for systems, where short response time is important (e.g. from the user experience perspective), those systems are preferable. 


\subsection{Discussion}

The evaluation shows that the benchmark provides a wide range of articulation through its parameter selection and the composition of the workload. However, despite the achieved configurability, the benchmark remains rooted in a real-life application and reflects the complexity of the implementation of such a system, which differenciates it from microbenchmarks. This connection to reality makes the conclusions drawn on the basis of the results more meaningful and applicable to practical use cases.

For example, given a specific application like a distributed data warehouse for use in analytics and decision support, where it is characteristic to expect predominantly read-only transactions that aggregate data from various sources, the evaluation data gathered in the evaluation indicates that in this particular use-case pessimistic TM would perform better on average than other types of concurrency control schemes (Fig.7d). However, we also note that this is the case only if the contention is fairly high. To the contrary, as the number of clients depreciates, an optimistic TM is the better choice to capitalize on low overhead. This data is therefore immediatelly useful to an architect involved in the design of such an application.

Another example is that of a geographically distributed system of sensors periodically checked by a network of servers, that use transactions to maintain consistency. Here, a large quantity of small read operations is expected as in Fig.7a. The case is similar to the one above, but while optimistic TM is significantly more performant in low contention, its throughput degrades in high contention, while pessimistic TM performs in a more stable fashion. This allows the architect to select either TM based on the variability of the server network.

On the contrary to both previous system types, the data from Fig. $7 \mathrm{f}$ shows that when designing a datastore used primarily for archivization, i.e. one with a large amount of long and write-oriented transactions, one need not bother with sophisticated synchronization schemes, since the number of conflicts involved forces near-serial execution in any case.

Alternatively, developers of TM systems can use the data provided by Helenos to analyze the systems and easily find their strong and weak points, whether inherent or ammeanable. For instance, as presented above HyFlow2 shows some room for improvement when subjected to write-heavy workloads with high retry rates. Knowing this, the developers of HyFlow2 can tune their contention management. They can still use Helenos for this purpose by creating some specialised scenarios for detailed examination of very specific situations and workloads. This can lead to the identification of the source of such behavior in the TM design. As for Atomic RMI, its notably worse performance in terms of MFT comparing to the HyFlow2 can be at least partially attributed to the fact that this system does not distinguish between read and write operations. Presumably introducing such distinction in the internal workings of this system and maximizing the parallelization of read operations can lead to closing the MFT performance gap between said TM systems.

\section{Conclusions}

We presented Helenos, a benchmark for performing comprehensive evaluations and comparisons of distributed TM systems. It provides depth of analysis by its implementation of a complex use case. It also allows for analyzing many aspects of the memory using a wide variety of parameters and metrics. Finally, it is based on a real application, meaning that the results of evaluations are more likely to be reflected in practice when the TM system is deployed in the real world. As such, it fills the vacuum caused by a lack of distributed TM benchmarks other than microbenchmarks.

We showed that Helenos provides important and meaningful data regarding TM system performance in near real-life situation. Helenos, in contrast to other benchmarks that have been used for testing distributed TMs, is built particularly for evaluating distributed TMs, and allows to do so with specific distributed applications in mind. Users of Helenos can use several, different parameters to create specialized scenarios to suit their specific needs and use them to perform extenvie and complex evaluation.

In our future work we wish to create a full suite of benchmarks for testing distributed TMs, to fulfill the requirement for broad evaluations, as well as deep ones. That is, in order to be able to evaluate distributed TMs in different applications from different fields. To that end we plan on distributing some applications from the STAMP 
benchmark suite, as well as to find additional applications that are specific to distributed systems. The work on these is already underway and the early results are promising.

Acknowledements The project was funded from National Science Centre funds granted by decision No. DEC2012/07/B/ST6/01230.

\section{References}

[1] M. Ansari, C. Kotselidis, K. Jarvis, M. Luján, C. Kirkham, and I. Watson. Lee-TM: A non-trivial benchmark for transactional memory. In Proceedings of ICA3PP'08: the 8th International Conference on Algorithms and Architectures for Parallel Processing, June 2008.

[2] V. Aslot, M. Domeika, R. Eigenmann, G. Gaertner, W. B. Jones, and B. Parady. SPEComp: A new benchmark suite for measuring parallel computer performance. In Proceedings of WOMPAT'01: the International Workshop on OpenMP Applications and Tools: OpenMP Shared Memory Parallel Programming, July 2001.

[3] R. L. Bocchino, V. S. Adve, and B. L. Chamberlain. Software Transactional Memory for Large Scale Clusters. In Proceedings of PPoPP'08: the 13th ACM SIGPLAN Symposium on Principles and Practice of Parallel Programming, Feb. 2008.

[4] J. C. Corbett and et al. Spanner: Google's Globally-Distributed Database. In Proceedings of OSDI'12: the 10th USENIX Symposium on Operating Systems Design and Implementation, Oct. 2012.

[5] M. Couceiro, P. Romano, N. Carvalho, and L. Rodrigues. D2STM: Dependable Distributed Software Transactional Memory. In Proceedings of PRDC'13: the 15th IEEE Pacific Rim International Symposium on Dependable Computing, Nov. 2009.

[6] R. Guerraoui and M. Kapałka. Principles of Transactional Memory. Morgan \& Claypool, 2010.

[7] R. Guerraoui, M. Kapałka, and J. Vitek. STMBench7: A benchmark for software transactional memory. In Proceedings of EuroSys'07: the 2nd ACM SIGOPS/EuroSys European Conference on Computer Systems, June 2007.

[8] M. Herlihy and J. E. B. Moss. Transactional Memory: Architectural Support for Lock-free Data Structures. In Proceedings of ISCA'93: the 20th International Symposium on Computer Architecture, pages 289-300, May 1993.

[9] S. Hirve, R. Palmieri, and B. Ravindran. HiperTM: High Performance, Fault-Tolerant Transactional Memory. In Proceedings of ICDCN'14: the 15th International Conference on Distributed Computing and Networking, Jan. 2014.

[10] S. Hong, T. Oguntebi, J. Casper, N. Bronson, C. Kozyrakis, and K. Olukotun. Eigenbench: A simple exploration tool for orthogonal tm characteristics. In Proceedings of IISWC'10: the IEEE International Symposium on Workload Characterization, 2010.

[11] T. Kobus, M. Kokociński, and P. T. Wojciechowski. Hybrid replication: State-machine-based and deferredupdate replication schemes combined. In Proceedings of ICDCS'13: the 33rd International Conference on Distributed Computing Systems, July 2013.

[12] A. Lakshman and P. Malik. Cassandra: a decentralized structured storage system. ACM SIGOPS Operating Systems Review, 44:25-40, Apr. 2010.

[13] C. C. Minh, J. Chung, C. Kozyrakis, and K. Olukotun. STAMP: Stanford Transactional Applications for MultiProcessing. In Proceedings of IISWC'08: the IEEE International Symposium on Workload Characterization, Sept. 2008.

[14] S. Mishra, A. Turcu, R. Palmieri, and B. Ravindran. HyflowCPP: A distributed transactional memory framework for C++, Aug. 2013. 
[15] D. Peng and F. Dabek. Large-scale incremental processing using distributed transactions and notifications. In Proceedings of OSDI '10: 9th USENIX Symposium on Operating Systems Design and Implementation, Oct. 2010.

[16] W. Ruan, Y. Liu, and M. Spear. Stamp need not be considered harmful. In Proceedings of TRANSACT'14: the 9th ACM SIGPLAN Workshop on Transactional Computing, Feb. 2014.

[17] M. M. Saad and R. B. Transactional forwarding: Supporting highly-concurrent STM in asynchronous distributed systems. In Proceedings of SBAC-PAD'12: the 24th IEEE International Symposium on Computer Architecture and High Performance Computing, Oct. 2012.

[18] M. M. Saad and B. Ravindran. HyFlow: A High Performance Distributed Transactional Memory Framework. In Proceedings of HPDC '11: the 20th International Symposium on High Performance Distributed Computing, June 2011.

[19] K. Siek and P. T. Wojciechowski. Atomic RMI: a Distributed Transactional Memory Framework. In Proceedings of HLPP'14: the 7th International Symposium on High-level Parallel Programming and Applications, pages 73-94, July 2014.

[20] K. Siek and P. T. Wojciechowski. Brief announcement: Relaxing opacity in pessimistic transactional memory. In Proceedings of DISC'14: the 28th International Symposium on Distributed Computing, 2014.

[21] K. Siek and P. T. Wojciechowski. Atomic RMI: A Distributed Transactional Memory Framework. International Journal of Parallel Programming, 2015.

[22] A. Turcu, B. Ravindran, and R. Palmieri. HyFlow2: A High Performance Distributed Transactional Memory Framework in Scala. In Proceedings of PPPJ'13: the 10th International Conference on Principles and Practices of Programming on JAVA platform: virtual machines, languages, and tools, Sept. 2013.

[23] P. T. Wojciechowski. Language Design for Atomicity, Declarative Synchronization, and Dynamic Update in Communicating Systems. Poznań University of Technology Press, 2007.

[24] S. C. Woo, M. Ohara, E. Torrie, J. P. Singh, and A. Gupta. The SPLASH-2 programs: characterization and methodological considerations. In Proceedings of ISCA'95: the 22nd Annual International Symposium on Computer Architecture, May 1995.

[25] F. Zyulkyarov, V. Gajinov, O. S. Unsal, A. Cristal, E. Ayguadé, T. Harris, and M. Valero. Atomic Quake: using transactional memory in an interactive multiplayer game server. In Proceedings of PPoPP'09: the 14th ACM SIGPLAN Symposium on Principles and Practice of Parallel Programming, Apr. 2009. 